\title{
IGLESIA Y REPOBLACIÓN. \\ LA RED PARROQUIAL DE LA TRANSIERRA EXTREMEÑA \\ $(1142-1350)$
}

\author{
JUAN LUIS DE LA MONTAÑA CONCHIÑA \\ Universidad de Extremadura
}

\section{SUMARIO}

1. Introducción.- 2. La parroquia en el contexto de la repoblación extremeña.- 3. El desarrollo de la red parroquial.- 4. Conclusiones.

\section{INTRODUCCIÓN}

La estructura eclesiástica extremeña está sujeta a un ritmo de crecimiento y organización estrechamente vinculado a los fenómenos de conquista y repoblación cristianas del territorio. El nacimiento y desarrollo de las células parroquiales como base sobre la que se articula la estructura eclesiástica sigue un ritmo desparejo a la ocupación del espacio extremeño pues el proceso de repoblación extremeño está caracterizado por su lentitud e irregularidad. La organización parroquial evoluciona con la consolidación de las estructuras poblacionales posteriores a las labores militares de recuperación del territorio'.

\footnotetext{
'Trabajo realizado con la ayuda a la investigación de la Fundación Caja de Madrid.

Juan Luis DE La MONTAÑa ConchiÑA, La Extremadura Cristiana (1142-1350). Poblamiento, poder y sociedad, Tesis de Doctorado leída en la Universidad de Extremadura. Inédita, Cáceres. 1998. Sobre cuestiones de repoblación y poblamiento medieval extremeño durante los siglos XIV y XV puede verse Angel BERNAL ESTÉVEZ, La repoblación del espacio extremeño en la Edad media: El poblamiento y la población, "Revista de Estudios

"Anuario de Estudios Medievales", 28 (1998)
} 
Una parte de las causas que determinan este desigual desarrollo hay que buscarlas en el desequilibrado proceso repoblador que se está proyectando en Extremadura desde los primeros años de siglo XIII. La labor repobladora y la coetánea estructuración de los marcos señoriales ${ }^{2}$ fueron los pasos previos a la conformación de una trama de obispados, arcedianatos y parroquias que imponían sobre la primera vertebración jurisdiccional laica una segunda malla no menos densa y compleja ${ }^{3}$.

La parroquia tiene una dimensión económico-señorial sobre la que se apoya el poder eclesiástico ${ }^{4}$. La circunscripción parroquial tiende a identificarse con la circunscripción laica y de algún modo son realidades compatibles que se superponen. Sobre el marco territorial de una villa 0 población rural se sobrepone el marco parroquial en un proceso dinámico de paulatina identificación estrechamente relacionado con la intensidad repobladora y el crecimiento demográfico de la población y de la comarca

Extremeños”, LI/3 (1995), pp. 627-645.

${ }^{2}$ Desarrollo de los marcos jurisdiccionales señoriales, concejos realengos, maestrazgos y encomiendas como células de organización del poder y control propia de las órdenes militares (José Vicente MATELLANES MERCHÁN, La Orden de Santiago y la organización social y económica de la Transierra castellano-leonesa (siglos XII-XIV), Tesis doctoral inédita, Universidad Autónoma de Madrid, 1996, pp. 556 y ss). También puede consultarse Juan Luis DE La Montaña Conchiña, Obispados y Ordenes Militares. Problemas jurisdiccionales en la Transierra extremeña del siglo XIII, "Revista Alcántara", 34 (enero-abril, 1995), pp. 29-48.

${ }^{3}$ Esta dinámica repobladora y conformadora de los marcos señoriales está claramente representada en las labores reconquistadoras y repobladoras desarrolladas en la expansión cristiana hacia el mediodía peninsular. En los momentos de máxima expansión territorial, la fundación de una sede episcopal siempre fue considerada por los monarcas como una base de partida para articular todo un proceso repoblador en torno a él, precisamente dentro de este sistema que articulan los marcos diocesanos la parroquia adquiere una parte importante de su verdadera dimensión. En este proceso repoblador se vieron implicados diversos representantes del poder eclesiástico y se desarrolló en una doble dirección, en la constitución, defensa y ocupación de lugares próximos a la frontera, y por otro lado, la actividad repobladora que algunos prelados desplegaron en el ámbito de sus diócesis. Casos conocidos como el del obispo de Toledo don Rodrigo, quien mantuvo una dura pugna en la colonización y extensión de la red parroquial en la zona castellanomanchega con la orden de Santiago. (Derek W. Lomax, El arzobispo Don Rodrigo Jiménez de Rada y la Orden de Santiago, "Hispania", XIX/LXXVI (1959), pp. 323-365). Véase también el trabajo de Hilda GRASSOTTI, En torno a las primeras tensiones entre las Ordenes Militares y la sede toledana. "Anales de Historia Antigua y Medieval", 17 (1972), pp. 115-169. Es igualmente interesante el trabajo de Enrique Rodríguez PICAVEA, Las Ordenes Militares y la Frontera. Contribución de las Ordenes a la delimitación de la jurisdicción territorial de Castilla en el siglo XII, Madrid, 1994.

${ }^{4}$ Quintín AldEA, La economía en las iglesias locales en la Edad Media y Moderna, "Hispania Sacra", XXVI/51-52 (1973), pp. 27-42. 
en la que se ubica ${ }^{5}$. Inicialmente estos espacios debieron ser amplios e imprecisos, la escasa población y la también escasa presencia de establecimientos religiosos así lo apuntan ${ }^{6}$. Sin embargo, esta situación cambió conforme se consolidaba el proceso de colonización. Esta realidad tiene una especial proyección en el caso de Extremadura y en la etapa propuesta. La estructura eclesiástica en Extremadura tiene un desarrollo cronológico que, al igual que en labores de colonización, lo sobrepasa. La iglesia extremeña, pese a sentar sus bases territoriales y jurisdiccionales en algunas ocasiones incluso con anterioridad a las grandes demarcaciones laicas, está inmersa en un desarrollo lento en su etapa organizativa ${ }^{7}$. La lentitud e incluso vaguedad con que se concretan los marcos diocesanos son una muestra evidente de esta dinámica. Sirvan como ejemplo los casos de Coria y Badajoz, obispados que conocieron una auténtica avalancha de problemas jurisdiccionales y tributarios protagonizados por las órdenes militares ${ }^{8}$.

Tendremos que esperar, por tanto, hasta finales del siglo XIII para constatar los primeros pasos de una iglesia sólidamente constituida y participativa en labores de repoblación cuyos resultados tangibles son la gestación y desarrollo de parroquias. La red parroquial extremeña se constituye tardíamente y este proceso se prolonga hasta bien avanzado el

${ }^{5}$ J.A. GARCÍA DE CORTÁZAR, La sociedad rural en la España medieval, Madrid, 1988, pp. $90-93$

${ }^{6}$ Es una de las primeras novedades que se producen en la constitución del entramado parroquial y su identificación con la comunidad (J.A. GARCÍA DE CORTÁZAR, La sociedad rural, ob. cit., pp. 90-91).

${ }^{7}$ Las dificultades fueron muchas y en algunos casos hasta muy avanzado el siglo XIII parece que no alcanzaron un desarrollo óptimo, pues eran demasiadas las penurias (Ortí BELmONTE, Episcopologio Cauriense, Cáceres, 1959, p. 20, "Notabiliti tuae notum fieri volumus quod Cauriensem Episcopum nobiscum duximus retinendum tum quia in Ecclesia, quae sibi commissa est, grave inopia, sicut accepimus").

${ }^{8}$ José Luis MarTín Martín, Los tiempos medievales, en "Historia de Extremadura", II, Badajoz, 1986, p. 366. Hace referencias al mismo problema en su trabajo Los obispos de Extremadura en la Edad Media, "Revista de Estudios Extremeños", XLVII/1 (1991), pp. 82-83. Se refiere al tema de la movilidad, en Badajoz, lógicamente permanecieron menos tiempo porque los peligros y los beneficios eran menores, quizá por eso la elección de canónigos del lugar sea mayor que en las demás de diócesis de Plasencia y Coria, donde la movilidad lógicamente era menor, especialmente en la de Plasencia. Con relación a la pérdida de lugares a manos de Ordenes Militares véase Juan Luis DE LA MONTAÑa CONCHI$\tilde{\mathrm{N}} \mathrm{A}$, Obispados y Ordenes Militares... art. cit., pp. 44-45. 
siglo XIV, debido, fundamentalmente, a la escasa organización de los marcos eclesiásticos y a la debilidad de las estructuras poblacionales 9 .

\section{LA PARROQUIA}

\section{EN EL CONTEXTO DE LA REPOBLACIÓN EXTREMEÑA}

La parroquia constituye la célula básica en la estructura de la geografía eclesiástica. Trasciende incluso al ámbito de lo religioso para influir en la organización civil del espacio, fundamentalmente en el marco del proceso de territorialización necesario para la obtención del diezmo y otros derechos, de ahí que destaquemos su importancia. La iglesia parroquial constituye una unidad que, por un lado faculta el desarrollo de la estructura poblacional dotándola de una cierto grado de cohesión y organización, mientras que al mismo tiempo garantiza la aplicación de la jurisdicción eclesiástica sobre hombres y bienes materiales, y con ella la seguridad de percibir el diezmo eclesiástico ${ }^{10}$.

Desde esta perspectiva, en la parroquia como unidad básica en la articulación del poder eclesial se integran una serie de elementos necesarios para su existencia, de ahí su importancia dentro de la estructura eclesiástica. En primer lugar, hay que recalcar la imagen de la iglesia como lugar de culto. No podemos olvidar las bases económicas y al clero local, de extremada importancia para la administración de los oficios, fieles y cura pastoral. Sin embargo, es preciso la delegación de determinadas funciones por parte de la jurisdicción episcopal para que la iglesia parroquial funcione como tal, ello es lo que le diferencia de la simple iglesia como lugar de culto. Estas funciones están básicamente representa-

\footnotetext{
${ }^{9}$ Los primeros datos sobre una iglesia sólidamente constituida en Extremadura son relativamente tardíos. Pueden consultarse al respecto José Luis Martín Martín, Las constituciones de la iglesia de Coria en 1315, "Miscelánea Cacereña", Primera serie, Cáceres, 1980, pp. 209-218. También es interesante el trabajo de $\mathrm{M}^{\mathrm{a}}$ del Sagrario BAY Santana, El obispado de Coria en los siglos XII-XV. Intereses y conflictos en su diócesis, Memoria de Licenciatura inédita, Cáceres, 1985.

${ }^{10}$ Léopold GENICOT, Comunidades rurales en el Occidente Medieval, Barcelona, 1993, p. 39. Idea igualmente reseñada brillantemente para otros espacios de la Corona de Castilla por Carmen DíEz HERRERA, La formación de la sociedad feudal en Cantabria, Santander, 1990 , pp. 202-205, especialmente p. 205. Esta misma idea de la parroquia como elemento intermediario entre señores y campesinos es recogida por Antonio FURIÓ y Ferran GARCía, El feudalisme medieval valencià: un assaig d'interpretació, "Debats", 5 (1981), pp. 33-42.
} 
das por la capacidad de poder administrar sacramentos, formalizar entierros y celebrar bautizos.

Para que las funciones con que eran dotados estos establecimientos religiosos se desarrollaran con normalidad se ponía al frente el cura párroco, vinculado directamente al obispo. El párroco era el encargado de percibir los beneficios parroquiales de los fieles contribuyentes" ${ }^{\prime \prime}$ En algunos casos, cuando se trataba sólo de una simple iglesia sin la categoría de parroquia, los fieles eran enviados a otras iglesias parroquiales. No es extraño pensar en una sencilla parroquia atendiendo a varios pueblos cercanos e incluso proyectando una labor comarcalizadora preferentemente en amplias zonas deficitarias de población.

La lentitud con que se conforma la red parroquial, como ya hemos señalado antes, está relacionada con las deficiencias repobladoras del territorio extremeño y organizativas de la iglesia en él localizada. De ello no cabe duda alguna. Casi podríamos señalar sin temor a exageración alguna que la constitución de parroquias en el caso extremeño es asíncrona al desarrollo del poblamiento, que existe una falta de simultaneidad entre ambas realidades como resultado de lo endeble de las poblaciones y de las dificultades organizativas de un clero escaso y no demasiado bien dotado económicamente ${ }^{12}$.

Un dato que puede ayudar a confirmar esta falta de sincronía es la dificultad de identificar el espacio parroquial con el de la comunidad en etapas inmediatamente posteriores al alejamiento de la frontera ${ }^{13}$. El primer paso para esclarecer toda esta problemática es rastrear el proceso de constitución de parroquias teniendo como punto de referencia las noticias repobladoras en las que aparecen asociadas menciones a establecimientos eclesiásticos. Para ello tenemos como punto de partida el creciente desarrollo de la actividad repobladora que se inicia en Extremadura desde la

\footnotetext{
"Realiza importantes sugerencias en un marco similar al extremeño donde es de destacar la contluencia de jurisdicciones distintas Enrique PICAvea-Matilla, Aproximación a la geografia eclesiástica del arcedianato de Calatrava (siglos XII-XVI), "Hispania Sacra", 43 (1991), pp. 735-773.

'Zna situación difícil que en general atravesaba la iglesia hispana del momento pero que de manera particular sin duda atravesaba la iglesia extremeña, cuestión a la que debemos sumarle el agravante impuesto por la permanencia durante casi un siglo de la frontera con los musulmanes (Peter LiHeHan, La iglesia española y el Papado en el siglo XIII, Salamanca, 1975). p. 366.

${ }^{13}$ Hipótesis expuestas por José Luis Martín Martín, Los tiempos medievales, ob. cit.,
} 
segunda mitad del siglo XII ${ }^{14}$. La iglesia parroquial adquiere protagonismo con el movimiento colonizador cristiano de nuevas tierras introduciendo en los cuadros de poblamiento una nueva jerarquía. Desde esta perspectiva es fácil vincularla a los distintos movimientos desbrozadores y también a la creación y consolidación de nuevos núcleos de poblamiento $^{15}$. El resultado de todo ello es que con el paso del tiempo se produce una convergencia o identificación del espacio de la comunidad con el espacio parroquial. A este respecto cabe señalar que la parroquia en el caso extremeño fue un elemento fundamental en el nacimiento o la maduración de las diversas comunidades.

Existen indicios para el caso extremeño que apuntan la puesta en marcha de esta realidad décadas después de la conquista cristiana. Comienza a producirse una identificación entre términos poblacionales y espacios parroquiales. Dicho de otra manera, parece que la expresión parroquia define realidades más concretas, es decir, circunscripciones más reducidas ${ }^{16}$. El crecimiento demográfico de algunas zonas y poblaciones paralelo a la consolidación del sistema parroquial y la maduración a la que antes nos referíamos se materializa en zonas donde es documentado un crecimiento poblacional estable desde finales del siglo XII y primera mitad del XIII.

Hacia 1257, obtenemos indicios en los que puede verse la identificación entre ambas realidades y el desarrollo de circunscripciones más reducidas en las iglesias alcantarinas de Sierra de Gata "la eglesia de Cadahalso con su comarca, e pertinencia" ${ }^{17}$. Noticias de naturaleza similar nos llegan en la misma fecha para referirse a las iglesias de "Valencia, en Esparragal, en Herrera, en Mayorga, en Piedrabuena, en Azagala", lugares en los que los alcantarinos tenían que dar "por procuraciones de

\footnotetext{
${ }^{14}$ Es un proceso constatado para el resto de Europa. Gabriel FOURNIER, La mise en place $d u$ cadre paroissial et l'evolution du peuplement, en "Cristianizzazione ed organizzazione ecclessiastica delle campagne nell'alto medioevo: expansione e resistenze", Settimane XXVIII, I, Spoletto, (1982) p. 499.

${ }^{15}$ Ibidem, p. 532. "Les nouvelles paroisses sont en rapport soit avec des défrichementes et la fondation des villes neuves, soit avec la multiplication des preuvres et des châteaux".

${ }^{16}$ J.A. García DE CORTÁZAR, La sociedad rural...ob. cit., p. 91. Señala que con el paso del tiempo las grandes circunscripciones parroquiales tienden a verse disminuidas para definir espacios y realidades más concretas.

${ }^{17}$ José Luis Martín Martín, Documentación medieval de la catedral de Coria, Salamanca, 1989, doc. 16. Confirmación de 1256, enero, Anagni, doc. 17.
} 
estas sobredichas iglesias y de quantas se hicieren en las comarcas o en las pertenencias destas yglesias sobredichas de Salor allende" ${ }^{18}$. La dinámica de creación e identificación de espacios/parroquias acorde con la dinámica colonizadora que venimos señalando se intuye con cierta claridad en el importante número de conflictos surgidos entre la iglesia cauriense y los freyres alcantarinos por el control de los distintos derechos parroquiales ${ }^{19}$.

Aunque esta realidad no se muestra totalmente definida al menos en una parte importante del territorio extremeño, tenemos datos sobre la dinámica delimitadora del espacio parroquial acorde con el crecimiento poblacional de zonas muy concretas. Así, en Badajoz, en 1271, surgieron problemas en la demarcación parroquial precisamente por la poca claridad de los términos aldeanos en los que debería basarse. Según señala un documento dado por el papa Gregorio X, se había producido una confusión entre los límites de las iglesias parroquiales de Badajoz y la catedral señalando que la delimitación se hiciese por personas del lugar "viris antiquis et prudentibus". Este particular suceso hay que relacionarlo con lo que señalábamos antes, con el aumento de la población y el ajuste del espacio parroquial ${ }^{20}$.

A modo de hipótesis, y puesto que el marco parroquial posee una proyección espacial en el territorio en cierto sentido similar al de las poblaciones, podríamos advertir que la conformación de los límites parroquiales tuvo un desarrollo cronológico vinculado al crecimiento poblacional y consolidación de la comunidad. Dicho de otra forma, la falta de

\footnotetext{
${ }^{18}$ Ibidem, doc. 16. Ignacio José de ORTEGA Y COTES, Bullarium Ordinis Militiae Alcantara, Madrid, 1759, pp. 91-93.

${ }^{19} \mathrm{Gracias}$ a las continuas disputas entabladas entre la iglesia cauriense y el maestrazgo alcantarino tenemos referencias ciertas sobre el proceso general de creación de parroquias en los dominios de la citada Orden (José Luis MARTín MARTín, Documentación medieval de la...ob. cit., dơc. 36, "que ningun clerigo cante ni sotierre de aqui adelante sin vuestra liçenzia e sih vuestro mandado que nos avemos en vuestro obispado").

${ }^{20}$ Juan SÖLANì DE FIgUeroA, Historia Eclesiástica de Badajoz, I parte, III, Badajoz, reed. 1929 , pp. 101-102, documento de 1272: "Discordias in futurum: tam cathedralis, quam aliorum parroquialium ecclesiarum praedictarum parroquias, quae nondum fuerant limitatae". Solano añade al respecto, p. 103, "se valio de hombres ançianos y prudentes conque sabiendo cada uno de los curas, los limites y terminos de su parroquia, cono seria con mas individualidad sus feligreses y podria con mas facilidad acudirles en sus necesidades y socorrerlos con los santos sacramentos de la yglesia".
} 
límites o su escasa concreción depende en cierto sentido del crecimiento de los núcleos poblados ${ }^{21}$.

Sin embargo, aunque tras el análisis de estas escasas noticias pudiéramos intuir la puesta en marcha de un creciente desarrollo de la red parroquial, surge un nuevo problema impuesto por las propias limitaciones de las fuentes a la hora de rastrear el proceso de conformación de la red: No todas las iglesias son parroquias. Aunque bajo una misma terminología se incluye a simples iglesias y parroquias, existen sustanciales diferencias. Ello debe ponerse, una vez más, en relación con la densidad demográfica de las poblaciones y de las comarcas donde se localizan. No todas las iglesias tenían "so rector" que celebraba los oficios e impartía los sacramentos. Muchas pequeñas poblaciones quizá dependieron en última instancia de centros parroquiales localizados en las poblaciones más importantes, de entre ellas las villas. Aunque bien es cierto que la práctica totalidad de estos asentamientos contaban con iglesias ${ }^{22}$.

Partiendo de esta realidad podemos comprobar el significado de ambos términos y tratar de distinguir entre la simple iglesia y la parroquia. Por noticias que nos llegan desde finales del siglo XII en las que se precisan los derechos diocesanos de los obispos extremeños el término iglesia posee una amplia significación donde con seguridad debían incluirse también las primeras iglesias parroquiales existentes en estos momen$\operatorname{tos}^{23}$. En este sentido, las fuentes de finales del siglo XII no muestran con la precisión suficiente la distinción entre ambas, cuestión que dificulta aún más si cabe la labor de desentrañar el proceso $^{24}$. En la totalidad de

\footnotetext{
${ }^{21}$ Sirva como ejemplo las iglesias que hacia 1235 iban surgiendo en los términos de Béjar: "fratri episcopo et dilectis filiis capitulo placentino e altera super eclesiis de Beiar ac eius termino et de Tornavacas et quibusdam aliis eclesiis et rebus allis quaestio verteretur" (Alonso Fernández, Historia ý Anales de Plasencia, Cáceres, 1952, pp. 47-48).

22José Luis Martín Martín, Documentación medieval, ob. cit., doc. 15 y doc. 16. Ignacio José de ORTEGA y COTES, Bullarium Ordinis Militiae, ob. cit., pp. 91-93.

23Ángel BARrios García; Documentación medieval de la catedral de Ávila, Salamanca, 1981, doc. 32, "Ea propter venerabilis in Christo fratre tuis iustis postulationibus grato concurrentes assensu, diocesanum ius, quod in secura, que ad culturam noviter est redacta, et in eclessiis in territoriis abulense, arevalense, almetense et securense positis tam cultis quam incultis habere disnosceris, sicut ad te iure canonico pertinet et in cunctis ecclesiis tue diocesis habes. salvis privilegiis apostolice sedis".

${ }^{24}$ Gervasio Velo y Nieto, Coria. Reconquista de la Alta Extremadura, Cáceres, 1956. p. 195, "confirmo vobis, et successoribus vestris in perpetuum decimam partem totius realengi Alcantaram, et omnium terminorum eius quam olim dedi Domino Suero, vestre ecclesie quodam Episcopo. Preterea mando et firmiter tenendum statuo, ne aliquis Ecclesias
} 
los documentos de esta época se hace mención de forma genérica a ecclesiis que debe aludir a los escasos establecimientos religiosos fueran o no parroquias ${ }^{25}$. El límite entre ambas realidades no queda del todo esclarecido y es posible que pudiéramos deducir la existencia de iglesias parroquiales en puntos donde poblacionalmente se documenta un importante crecimiento. Tal es el caso, por ejemplo, de los valles placentinos donde se datan los primeros asentamientos permanentes (Oliva, Segura de Toro $)^{26}$.

A comienzos del siglo XIII la situación comienza a clarificarse. Según indicios que tienen que ver con el crecimiento poblacional se detecta la creación de las primeras parroquias. Es en estos momentos cuando se documentan los primeros intentos de percepción del diezmo, elemento común a la totalidad de las iglesias sean parroquiales o no. Las constituciones de Plasencia (1229) son claras al respecto, pues los derechos de bautismo y entierro (además de poder oficiar matrimonios y practicar la unción de los enfermos) sólo eran ostentados por aquellas iglesias más importantes, es decir, las parroquias a las que las constituciones aluden indirectamente. Estos indicios se confirman cuando apuntan el reparto de las raciones en las parroquias, o la recaudación por parte del arcediano de las procuraciones en las iglesias de la villa y aldeas dependientes de la catedral $^{27}$. Con todo, parece existir un primer indicio que permite distinguir a las primeras parroquias de las simples iglesias, más abundantes

que in Cauria, vel Alcantara, vel Alconetar, vel Caceres vel earum terminis".

${ }_{25}^{25}$ osé Luis Martín Martín. Documentación medieval, ob. cit., doc. 4, Bula de Lucio III al obispo de Coria don Arnaldo y sus sucesores, señala los límites de la diócesis y la confirma todas sus propiedades y privilegios, "Locum ipsum in quo praedicta Ecclesia sita est cum omnibus pertinentiis suis D. episcopatur tui. sicut defluunt aquae versus Cauriam per sumitates montium qui sunt a monte Salama, usque ad illos, qui sunt ultra Portum Munioz. quosque sunt oppositi Caparrae, ius diocesanum in Capara et terminis suis, et omnibus ecclesiis in Caceres et terminis suis, et omnibus eclesiies in Milana, Trebellio, Almenarella, Palumbaria, Ranconada, Atalaya Pelagii Vellido et terminis suis".

${ }^{26}$ Julián Clemente Ramos y Juan Luis DE LA MONTAÑA CONCHiña, La Extremadura cristiana (1142-1230). Ocupación del espacio y transformaciones socioeconómicas, "Historia, Instituciones, Documentos”, 21 (1994), p. 96. Diversas opiniones sobre la antigüedad de los lugares citados pueden encontrarse en Domingo SÁNCHEZ LORO, Historias placentinas inéditas. Catalogus episcoporum ecclessiae placentinae, I A. Cáceres, 1982. p. 414.

${ }^{27}$ Domingo SÁNCHEZ LORO, Historias placentinas inéditas, ob. cit.. p. 380. “Los canónigos que agora han raciones en las iglesias parroquiales, en la villa o en las aldeas. hayanlas en su vida y reciban enteramente las raciones si las quisieren servir en las iglesias así como los otros clérigos de esas mismas". 
debido a la multitud de pequeñas pero endebles poblaciones que comenzaban a salpicar los campos altoextremeños.

Avanzado el siglo XIII existen indicios claros a través de los cuales podemos distinguir la simple iglesia y la iglesia parroquial que goza de mayores atribuciones. Este hecho puede observarse con nitidez en las primeras constituciones de la iglesia pacense redactadas en 1255 "otrossi el aldeano pague la meatat de los diesmos quella eglesia de la aldea do labra e cria, e la otra meatat a la eglesia donde reçibe los sacramentos eclesiasticos" 28 . Se funden, por tanto, dos realidades importantes, la administración de sacramentos y la percepción de una parte de los derechos eclesiásticos - no sólo el diezmo- percibidos de las pequeñas iglesias sometidas a la jurisdicción parroquial y mantenidas por un número de clérigos muy reducido. El citado sínodo pacense de 1255 viene a confirmar esta realidad en la que no todas las iglesias por el hecho de desarrollar funciones recaudatorias ostentaban funciones parroquiales. Para ello se especifica que los pobladores que murieran en el término o en las iglesias de Valdesevilla, Revueltados, Albuera y Talavera - iglesias que sí podían recaudar el diezmo- debían enterrarse en la que fue iglesia catedral. Esta situación nos llega refrendada en las constituciones de la misma iglesia de 1279 , cuando señala que "no pongan capellanes e resçiban los sacramentos los que hi moran de los cureros de la see" 29 .

Como hemos podido comprobar no todas las iglesias gozan del estatus parroquial. Existe una diferencia neta entre la simple iglesia y la parroquia. Esta cuestión nos hace intuir que el proceso de constitución de las parroquias es lento y bastante más complejo, y debe relacionarse con la secuencia repobladora y el peso demográfico de los distintos lugares.

\footnotetext{
${ }^{28}$ Juan SolANO DE FIGUEROA, Historia Eclesiástica, ob.cit., I parte, III, pp. 14-15. Así se señala en las constituciones de Badajoz de 1255.

${ }^{29}$ Ibídem, I parte, III, pp. 12 y ss; y p. 125. "Non faga el obispo raçión prestamera nin servidera en las eglesias de Entelena con finojales, nin de cornudiella con mediniella, nin de la torreçilla con el carpio, ini del verçeal con malpartida nin de Botova, con covillana, porque son logares de la limitación de la see, nin ponga capellanes".
} 


\section{EL DESARROLLO DE LA RED PARROQUIAL}

Es factible pensar que el proceso seguiría unas pautas determinadas acorde con el acrecentamiento poblacional de Extremadura a partir de la segunda mitad del siglo XIII. El esquema en sus líneas generales es sencillo. Inicialmente, con el núcleo de poblamiento nacía una pequeña iglesia, o ésta servía como centro aglutinador de lo que posteriormente sería la aldea. Sólo la consolidación demográfica del lugar podía convertir en parroquia un pequeño centro eclesiástico o simple iglesia. Esta parroquia haría las funciones de eje vertebrador de la autoridad eclesiástica orientada a la percepción de diezmos y especialmente otros derechos en amplias zonas.

El modelo de esta realidad en el que el desarrollo de la parroquia se muestra como un proceso tardío y se identifica con el crecimiento y consolidación de la población, nos llega ejemplificado a través de la creación de la parroquia de Miajadas en 1348: "veyendo en como el pueblo de Meajadas, termino de Medelin, que es en nuestro obispado se acrescienta de cada dia gracias a Dios ordenamos y tenemos por bien que, de aquí adelante que haya iglesia bautismal en el dicho logar de Meajadas. Y tenemos por bien que el rector, que es agora en el pueblo del Escorial, que haya so cura y so rectoria en el dicho pueblo de Miajadas" ${ }^{30}$.

$\mathrm{La}$ información que proporciona este caso es fundamental para entender un proceso que debió abrirse en el siglo XIII y de cuya proyección zonal nos ocupamos a continuación. En el ejemplo de la parroquia fundada en la aldea de Miajadas vemos con claridad que la creación de este centro está vinculada a la importancia demográfica del lugar. El crecimiento de algunas poblaciones convirtió las pequeñas iglesias en centros parroquiales sobre todo si estos asentamientos -como es el caso de Miajadas- por su localización espacial y accesos presentaban las condiciones idóneas. Es posible pensar, que de esta forma centros poblacionales más densamente poblados se transformaban en pequeños ejes comarcalizadores de una dinámica repobladora y de poderosos intereses eclesiásticos.

En el caso de Coria no tenemos referencias ciertas sobre el organigrama parroquial de este obispado. Las disputas mantenidas ente alcantarinos y el obispo de Coria por las iglesias y sus derechos nos proporcio-

\footnotetext{
${ }^{30}$ Domingo SÁNCHEZ LoRo, Historias placentinas inéditas, ob. cit., II, pp. 232-233.
} 
nan indicios sobre su lenta constitución. Según la documentación, bajo el término ecclesiis seguramente se incluyen iglesias parroquiales en las que tanto el obispo como la orden dirimían sus respectivos derechos ${ }^{31}$. El desarrollo de la estructura parroquial debió comenzar a adquirir visos de consolidación en diversos puntos mencionados repetidas veces (Alcántara, Valencia de Alcántara y Santibáñez de Máscores). En un documento fechado en 1235 son mencionadas las iglesias de San Pedro en el castillo de Santibáñez el Alto, Portezuelo, Moraleja, Milana, Malladas y Piedras Albas $^{32}$. Es posible pensar que, como hemos venido señalando hasta el momento, este número reducido de iglesias en lugares de importancia poblacional como los reseñados señale la existencia de las primeras parroquias constituidas frente al resto de las iglesias localizadas en pequeñas fortificaciones y asentamientos aldeanos. Un dato más exacto sobre esta realidad nos llega en 1294. La orden alcantarina reconoce al obispo de Coria una serie de derechos que atañen a la actividad parroquial. Tal es así, que los clérigos nombrados por la orden en las iglesias "de la nuestra tierra que nos avemos en vuestro obispado" no podían realizar enterramientos ni "cantar" sin permiso del obispo o sus vicarios ${ }^{33}$.

Empero, si observamos los datos documentales, las noticias aportadas son ambiguas y no permiten ver con la claridad deseada el desarrollo parroquial. Referencias tan generales como las señaladas y otras más concretas aluden a la lenta organización eclesiástica de la diócesis de Coria. Es sintomático el hecho de no tener noticias de los tres arcedianatos (Coria, Galisteo y Cáceres) en que se dividía la diócesis hasta comienzos del siglo XIV, y hasta 1315 no se conocen las primeras constituciones para la iglesia de Coria ${ }^{34}$.

A falta de noticias, el desarrollo y territorialización de los arcedianatos puede ayudar a explicar esta realidad. Las primeras noticias que nos llegan de los arcedianos de Plasencia datan de las primeras décadas del siglo XIII, síntoma evidente de un intento de organización de la iglesia. Tenemos constancia de arcedianatos en las villas de Béjar, Trujillo y

\footnotetext{
${ }^{31}$ José Luis Martín Martín, Documentación medieval, ob. cit., doc. 36.

${ }^{32}$ José Ignacio ORTEGa Y COTES, Bullarium Ordinis Militiae, ob. cit., scrip XVIII, pp. 42-45.

3.josé Luis Martín Martín, Documentación medieval, ob. cit., doc. 36; José Ignacio ORTEGA Y COTES, Bullarium Ordinis Militiae, ob. cit., p. 73.

${ }^{34}$ José Luis Martín Martín, Documentación medieval, ob. cit., doc. 73.
} 
Medellín, al frente de los cuales debieron situarse otros tantos arcedianos. Es probable que, vistos estos datos, comenzaran a desarrollarse las primeras parroquias ${ }^{35}$.

Si en un plano superior la iglesia placentina fijaba rápidamente sus estructuras, en el plano local esta realidad debió tener una proyección similar aunque variable lógicamente en función de la población de las distintas comarcas. Con el fin de distinguir las simples parroquias del resto de establecimientos religiosos, las constituciones placentinas ya aluden a "iglesias parroquiales" al frente de las cuales estaban los párrocos. Por contra, en el otro extremo se encuentran las simples iglesias o "iglesias aldeanas" como las definen en las fuentes cuando se refieren sobre todo al ámbito rural ${ }^{36}$.

Noticias fechadas en 1254 pueden corroborar nuestras ideas. En la confirmación de los estatutos de la iglesia que hizo el Papa Inocencio IV se hace una relación de iglesias y lugares, de entre los cuales hay que destacar las aldeas "de Xarahiz, de Quacos, de Jarandella" donde el obispo y cabildo se reservan una ración debido a las escasas rentas decimales del resto de las iglesias ${ }^{37}$. Esto nos hace pensar en que seguramente eran las aldeas que más contribuían económicamente. Quizá la importancia poblacional y económica de las mismas las situó como primeras parroquias en el organigrama del obispado placentino ${ }^{38}$. Además, son citadas junto a las iglesias parroquiales de la ciudad y de las villas integradas en el obispado las de Trujillo, Béjar y Medellín ${ }^{39}$.

En relación con los aspectos que estudiamos, el obispado de Badajoz nos proporciona datos interesantes que pueden ayudar a clarificar y sintetizar el proceso. El escaso peso demográfico de algunas de sus pobla-

\footnotetext{
${ }^{35}$ Domingo SÁNCHEZ LORO, Historias placentinas inéditas, ob. cit., vol. A, pp. 382-383 En las constituciones placentinas de 1229 son mencionados los arcedianos de Plasencia y Béjar.

${ }^{36}$ Vid. nota anterior, y p. 392, en el documento de 1254 que es una Bula del pontífice Inocencio IV se confirman específicamente los estatutos de esta santa iglesia catedral y es mencionado el arcediano de Trujillo junto al de Plasencia, sin embargo, no se hace referencia al de Béjar, el cual se conoce desde 1229.

${ }^{37}$ Ibídem, p. 380. "Cuando el arcediano fuere a alguna iglesia de las aldeas a tomar procuración, vayase, salvo si alguno se lo quierere dar de grado. Y si alguna iglesia no pudiere abastar, ayunten una o muchas, que puedan sin agravamiento".

${ }^{38} \mathrm{ACP}$, Sección Bulas, leg. II, doc. 6.

${ }^{39}$ Domingo SÁNCHEZ LoRO, Historias placentinas inéditas, ob. cit., vol. B, p. 408.
} 
ciones y el lento desarrollo de la institución eclesiástica en este concejo debió repercutir en el desarrollo de la estructura parroquial. Las iglesias no parroquiales dependientes de la catedral citadas en 1255 habían aumentado sensiblemente en 1279 como señalan las constituciones. Allí se localizan las iglesias de Entelena, Hinojales, Cornudiella, Mediniella, Torreçilla, Carpio, Verçeal, Malpartida, Botova, Covillana, dependientes de la catedral $^{40}$. Por contra, el número de parroquias creció en menor cantidad, alcanzado tal rango aquellas que se localizaban especialmente en poblaciones de mayor importancia demográfica, iglesias curiosamente alejadas del centro rector y anteriormente en posesión de órdenes militares.

En este contexto se documenta la incorporación de una serie de lugares a partir de 1284 que, como decíamos anteriormente, fueron lugares incluidos en las jurisdicciones de las órdenes militares de Santiago y el Temple. Entre estas poblaciones se encuentran Olivenza, Táliga, Los Santos, Villanueva (de Barcarrota) y Aldea de los Caballeros. Afortunadamente se han conservado los documentos en los que se constata la toma de posesión de las respectivas iglesias por el obispo y la constitución como parroquia. De entre estas poblaciones citadas Olivenza constituye el ejemplo a seguir porque casi con seguridad era la más poblada ${ }^{41}$.

En el acto fundacional de la parroquia se tomaba "posesión pacífica" de la iglesia en cuestión para luego colocar al frente de ellas los curas racioneros. El obispo retenía para sí la tercia pontifical del total del diezmo recaudado. El resto del protocolo queda perfectamente detallado en el documento: "Martin Sanchez su chaçeler, e su criado, que estaba hi presente, et envestiolo luego dellas por su anillo doro que traia, metiendogelo en el dedo de la diestra mano, et levantose de un tabet enque se ia posado, e tomó al dicho Martin Sanchez por la mano e metiolo e instituyolo corporalmente en la dicha eglesia por Prior e Retor deela, et por Raçionero. et fiço antel pueblo del logar que daquel dia en adelante a el catasen e

\footnotetext{
${ }^{40}$ Juan Solano De Figueroa, Historia Eclesiástica, ob. cit., I parte, III, p. 12 y ss.

${ }^{41}$ Un dato más que avala esta cuestión es que en la iglesia de Olivenza se hacen hasta cinco raciones para los clérigos, mientras que en el resto se hacen tres y una ración, síntoma de la pobreza del lugar y por tanto de su escasa población (Juan Solano DE FIGUEROA, Historia Eclesiástica, ob. cit, I parte, III, p. 150 y ss).
} 
reçibiesen por Prior, e por su Rector, e del reçibiesen los sacramentos todos de Santa eglesia, e non de otro ninguno"42.

Para el resto de las poblaciones incorporadas a la jurisdicción episcopal no se hace referencia alguna a la probabilidad de administrar sacramentos, sólo se señala la posibilidad de recaudar diezmos y primicias en las poblaciones de Los Santos y Villanueva, poblaciones menos importantes -demográficamente hablando- que Olivenza ${ }^{43}$. Para aseverar esta realidad, según los distintos documentos, las dotaciones en clérigos, utensilios eclesiásticos, ropas y riquezas son menores. De esta forma, podemos aventurar la conformación de una trama parroquial que tiene en las poblaciones de mayor densidad poblacional el eje central en torno al que se organizaría una red de pequeñas iglesias de ella dependiente.

\section{CONCLUSIONES}

La lenta repoblación del territorio extremeño tiene como consecuencia un asentamiento poblacional insuficiente en la mayoría de los casos, rebosante de contrastes por cuanto confluyen zonas de intenso poblamiento con zonas prácticamente deshumanizadas. También se detecta una eficiente articulación de los marcos de poder señoriales. La red parroquial en la Extremadura cristiana de entre 1142 y 1350 está sujeta a este desarrollo lento y desigual de la estructura eclesiástica en general y de la poblacional en particular.

En primer lugar no podemos dejar de señalar la existencia de una iglesia extremeña atravesando grandes dificultades. La permanencia de la frontera no favoreció en absoluto su rápida extensión ni organización territorial. Sólo con posterioridad al alejamiento de la misma se percibe una organización más estable. Se conocen las primeras constituciones catedralicias (Plasencia 1229, Badajoz 1255), se organizan los primeros arcedianatos y con todo ello se intuye un primigenio desarrolla de la red parroquial.

En este primer momento las escasas referencias y la imprecisión de las fuentes sólo permiten detectar la existencia de establecimientos

\footnotetext{
${ }^{42}$ Ibídem, p. 151.

${ }^{43}$ Ibídem, I parte, III, pp. 157-159.
} 
religiosos bajo expresiones tan comunes como eclesiis o eglesias. Datos directos sobre la identificación existente entre el espacio de la comunidad y el espacio parroquial comienzan a documentarse en torno a 1257 en las comarcas más pobladas (Sierra de Gata, valles placentinos, alfoz de Badajoz). Con todo, la organización parroquial sigue presentándose muy difuminada.

El crecimiento poblacional que se documenta en Extremadura a finales del siglo XIII y comienzos del XIV es el inicio de una realidad distinta. Constatamos la lenta conformación de establecimientos parroquiales en aquellos lugares en los que el crecimiento poblacional parece tener mayor proyección. Los primeros datos nos llegan hacia 1284 para el caso de Badajoz. En 1284 se constituye una parroquia en la población de Olivenza, y se toma posesión de otras iglesias. En términos de Coria el proceso se presenta más oscuro, pero hacia 1294 parece que esta dinámica está puesta en marcha. En el alfoz placentino desde 1254 se documenta esta realidad alcanzando mayor significación en la primera mitad del siglo XIV. Quizá el citado documento de 1348 en el que se constituye la parroquia de Miajadas como resultado de un importante crecimiento de la población sea el mejor ejemplo y síntesis de la totalidad del proceso.

\section{RÉSUMÉ}

Le réseau paroissial d'Estrémadure se caractérise par son lent développement. Une fois supéré le procès de la conquête du territoire, il ne sera qu'à la fin du XIII' siècle que la structure paroissiale acquerra une certaine apparence de développement, lequel est conditionné basiquement par une double réalité. En premier lieu. il faut signaler l'organisation ecclésiastique, toujours déficiente, dans un espace géographique plein de difficultés et une lente colonisation. En deuxième lieu, on doit considérer l'extrême pénurie de poblation que présentaient beaucoup de contrées d'Estrémadure très avancé le XIII" siècle. Sans aucun doute, l'inconsistente réalité paroissiale d’Estrémadure, visible jusqu'au début du XIV ${ }^{\mathfrak{c}}$ siècle, est très attachée à un procès de repeuplement insufisant et d'intense localisation dans lequel on détecte d'énormes contrastes entre des zones ocupées et des zones pratiquement dépeuplées. Au début du XIV siècle, on a affaire à une organisation du réseau paroissiale plus forte. le résultat évident d’une croissance généralisée de la population dans la totalité du territoire d'Estrémadure. 


\section{SUMMARY}

The parish network of Extremadura can be characterised by its slow development. Once the conquest of the territory had taken place, it would not be until the end of the 13th century that the parish structure would acquire a certain development. This development basically would rely, in essencee, on two realities. Firstly, we must point out the still inadequate ecclesiastical organization in a space full of difficulties and slow colonization. Secondly, the extreme sparcity of the population in many of the regions of Extremadura by the middle of the 13 th century should be taken into consideration as well. Without a doubt, Extremadura's reality of a weak parish system. evident until the start of the 14th century, is closely related to an insufficient repopulation process and to an intense localization in which major contrasts can be seen between settled zones and practically uninhabited zones. At the beginning of the 14th century, a better parish network organization can be seen as the evident result of a general population increase in the totality of the territory of Extremadura. 